Original scientific paper

\title{
SIMULATION BASED RESOURCE CAPACITY PLANNING WITH CONSTRAINTS
}

\author{
Ojstersek, R. \& Buchmeister, B. \\ University of Maribor, Faculty of Mechanical Engineering, Smetanova 17, 2000 Maribor, Slovenia \\ E-Mail: robert.ojstersek@um.si, borut.buchmeister@um.si
}

\begin{abstract}
The research work represents the development of a new decision-making model intended for the resource capacity planning depending on the production system constraints. A mathematical decision model for medium and short-term (dynamic) workers allocation was developed. A simulation study of the dynamic events and the response of the decision-making algorithm to achieve the optimal workers allocation was conducted. The results show that the resource capacity planning has a high importance as the constraints faced by workers in production systems become more severe. The results demonstrate the high ability to terminate production capacity while ensuring smooth, efficient operation of the production system. Presented method can be used in everyday workers allocation in different types of production systems.

(Received in August 2021, accepted in November 2021. This paper was with the authors 1 month for 2 revisions.)
\end{abstract}

Key Words: Simulation Modelling, Mathematical Modelling, Resource Capacity Planning, Constraints Theory, Decision-Making Algorithm

\section{INTRODUCTION}

Regardless of the production systems type, especially in developed countries, we are dealing with a shortage of suitable workforce. The shortage of adequate workforce capacity and proper allocation of available workers are critical to ensure continuous operation of production systems. In practice, workers planning and scheduling is carried out from two temporal perspectives. The first and most used is cyclical medium-term planning of available workers for a few weeks and months in advance. This type of planning does not pose an excessive problem from the planner's point of view if he/she has enough personnel and known production system resources. However, in real life there are daily problems due to the absence or restriction of individual employees. In this case, these are dynamic events that occur all the time. In smaller production system, the planner can reallocate personnel and at the same time ensure satisfactory (not optimal) operation of the production system, but when we talk about larger production systems, it is impossible to solve this satisfactorily.

Researchers have been working for many years to develop successful and efficient planning and scheduling of employees to ensure employee satisfaction and successful operation of the production system $[1,2]$. In companies, they strive for the optimization parameters to achieve the expected due dates [3]. Efficient operation of the production system is the goal of the company [4], which must also achieve employee satisfaction while ensuring partial flexibility [5, 6]. Accommodating all the individual needs of employees is theoretically impossible [7], but recently more and more employees are emerging who have individual constraints that the company must accommodate [8]. Scheduling workers with different constraints in different jobs while ensuring minimal losses is a major challenge [9], so in this case researchers use scheduling of different workers on identical machines or heterogenous knowledge $[10,11]$. Scheduling workers using simulation modelling approaches [12] is primarily related to the use of a specific programming environment [13]. Given the limitations of simulation models, the recent application of artificial intelligence methods with various numerical optimization techniques developed by researchers, the optimized operation of production process is enabled [14-16]. However, the objectives of scheduling appropriate for work shifts [17], tasks [18], and 
providing adequate knowledge to workers [19] are present. If we talk about medium-term cyclic planning, which is a less challenging problem in terms of optimization, dynamic events are more challenging from the perspective of optimal planning, as they are limited examples of complex problems $[20,21]$. Inadequate capacity planning has a negative impact not only on pre-ordering as a whole $[22,23]$, especially when it comes to underutilization of automated production systems [24].

In this research paper, we aim to present a contemporary mathematical decision-making using the algorithm that classifies workers according to the type of competence and the constraints of workers to the available production capacity. The main research contribution is a decision-making model capable of medium and short term (dynamic) allocation of workers with constraints. The developed algorithm must provide flexibility and the possibility of application to complex decisions of managers.

\section{RESOURCE CAPACITY PLANNING}

Resource capacity planning (RCP) is the process of defining the amount of work that can be done with the available resources at a company, determining the tasks that can be done by allocating resources appropriately, and matching the work to be done with the available resources to meet current and future needs. If the company is able to understand the current and future available capacity and prepares detailed resource plans, it can make the most of its resources and justify the system. Resource capacity requires a thorough understanding of resource capabilities (workers, machinery, equipment, etc.), the requirements of each task performed by a company, realistic schedules for tasks, and an effective short-, medium-, and long-term resource strategy. When resource capacity planning is optimized, it has a great positive impact on a company. Resource capacity needs to be calculated so that managers (production unit, shift, etc.) have a better understanding of their team's capabilities and resources.

Some general formulations of the basic RCP parameters can be made:

- Determine the total number of hours worked in the period measured/used.

- Determine the net hours worked using appropriate number of workers.

- Determine the individual capacity for each resource by evaluating the team members for the time period used.

- Combine the individual capacity of each resource to determine the total hours for the team capacity.

An accurate RCP model should include details about the types of work (from a technology perspective) in the task lifecycle and the specific skills (from a worker perspective) required to complete each [25]. This makes it possible to understand who should be assigned to different tasks based on their skills. Appropriate allocation can help to better control tasks and track time during the exact implementation of the task. Effective RCP uses skill matching to make the best use of available resources. Skill matching uses a database that compiles correlations to individual available resources with a detailed task and knowledge assessment. These insights help company quickly understand the strengths and capabilities of individual resources so that an efficient RCP can be ensured. Skill matching helps a company understand the capabilities its resources currently provide, as well as which resources may need to be added and which type of training is required to achieve greater depth of competency either by hiring full-time staff or outsourcing resources.

In the time of Industry 4.0 and collaborative workplaces, resource specialization is becoming an increasingly important element of successful resource management. Companies today expect more specialized knowledge and skills that require unique capabilities from resources. Effective RCPs must have and leverage a skills database to keep track of which team 
members have which knowledge and skills, better have those specialized skills in record, so they can be assigned appropriately when needed. In addition, RCP models must also account for outsourcing resources when accessing unavailable skills, knowledge, or capacity. Having a robust RCP approach, these skills are always available to help at the company's success.

High resource utilization depends on the ability to allocate a resource from task to task with as little unnecessary idle time as possible. Successful RCP makes this possible and ensures high, sustained utilization for the business by matching resources, their capabilities, and the demands of the tasks. As companies continue to implement their RCP strategy, data from previous RCPs creates a deeper catalogue of resource capabilities and capacities to further improve planning and resource matching. These incremental improvements can have an increasingly positive impact on utilization rates until a company reaches a level of utilization that makes it profitable while preventing employee burnout. Fig. 1 presents the proposed RCP organization diagram for workers allocation in a manufacturing system.

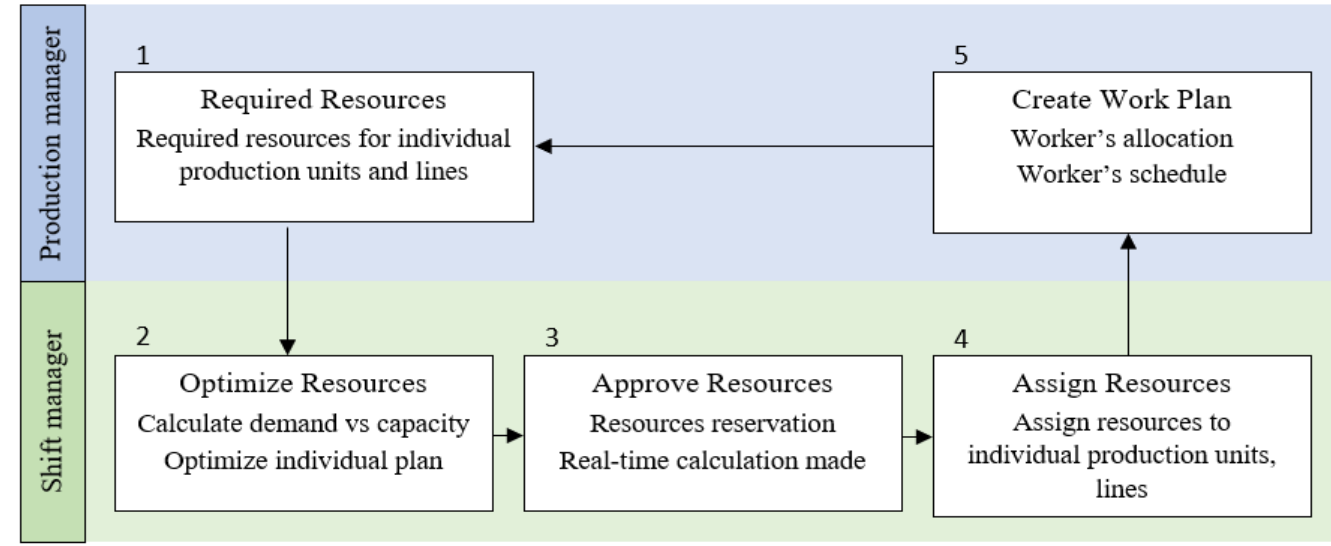

Figure 1: Proposed RCP diagram.

\subsection{System description}

In the research problem under consideration, we want to optimally schedule three types of workers according to their knowledge and the limitations we may have. The main condition is the uninterrupted operation of the production system, which must always operate smoothly. The present case concerns the mass production of product packaging. The research problem is based on the development of a decision model for determining the optimal number of workers according to the needs and available resources. Table I shows the input parameters of the production unit, the number of automated lines, their name (designation), the minimum number of workers of a specific type (mechanics, operators, and technicians) and the number of shifts in which the production lines operate.

Presented decision-making algorithm addresses three Production Units (PUs). All PUs operate in 3 shifts, 5 days per week, on 22 automatic lines. Total number of production workers is 217. Each PU presents unique entity with different demands and structure of employees. For those reasons it is difficult to determine the optimal shift conditions for each employee. Goal of the research work is to determine the maximal number of employees that can change shift mode, without significant impact on productivity (taking into account $100 \%$ lines productivity).

Additional decision-making constraints under the consideration:

1. Metaheuristic decision-making method for creating a model for determining maximal number of employees that production can afford to change shift model with different workers' limitations.

2. Model is suitable for three production types of workers' shift allocation: operators, mechanics and technicians. 
3. Shift organization model does not include manual packaging lines, manipulators, and shift managers.

4. Decision-making model is created in MatLab environment, input parameters and numerical results are transferred from Excel to MatLab and vice versa.

Table I: Production unit requirements.

\begin{tabular}{|c|c|c|c|c|c|c|}
\hline \multirow{2}{*}{ Line no. } & \multirow{2}{*}{ Line name } & \multicolumn{4}{|c|}{ Minimum number of workers } & \multirow{2}{*}{ No. of shifts } \\
\cline { 3 - 6 } & & Mechanics & Operators & Technicians & $\sum$ & \\
\hline 1 & A 13 & 1 & 1 & 0.5 & 2.5 & 3 \\
\hline 2 & A 23 & 1.5 & 1 & 0.5 & 3 & 3 \\
\hline 3 & A 22 & 1.5 & 1 & 0.5 & 3 & 3 \\
\hline 4 & A 17 & 1 & 1 & 0.5 & 2.5 & 3 \\
\hline 5 & B 87 & 1.5 & 1 & 0.5 & 3 & 3 \\
\hline 6 & B 88 & 1 & 1 & 0.5 & 2.5 & 3 \\
\hline 7 & B 07 & 1 & 1 & 0.5 & 2.5 & 3 \\
\hline 8 & B 78 & 1 & 1 & 0.5 & 2.5 & 3 \\
\hline
\end{tabular}

Table II shows the input parameters of PU1, showing the current values of available mechanics, operators and technicians in individual shifts. It should be emphasized that the number of available workers is constantly changing, as the fluctuation in production is large, as well as workers are constantly being educated and changing positions. All these changes must be addressed by the decision-making model.

Table II: Number of workers in the next default schedule.

\begin{tabular}{|c|c|c|c|c|}
\cline { 2 - 5 } \multicolumn{1}{c|}{} & Number of mechanics & Number of operators & Number of technicians & $\sum$ \\
\hline Shift A & 8 & 13 & 6 & 27 \\
\hline Shift B & 10 & 11 & 5 & 26 \\
\hline Shift C & 10 & 11 & 5 & 26 \\
\hline$\Sigma$ & 28 & 35 & 16 & 79 \\
\hline
\end{tabular}

\subsection{Definitions of constraints}

Mathematical decision-making model is made according to the next constraints:

1. Each packaging line is operated by an operator, a mechanic, and a technician.

2. Minimum standard of workers per line is 1 mechanic, 1 operator, 0.5 technician. Some lines due to complexity require additional personnel.

3. Employee can move between shifts, inside its own production unit.

4. Employee can move between packaging lines in his/her unit (except in unit 2 where there are 2 types of packaging lines, the condition is also knowledge of mechanics regarding the packaging line characteristics).

5. If needed, technician can replace operator. Other replacements are not allowed.

6. European low requirements, regarding working conditions, must be respected.

Mathematical decision-making model uses the following variables under the consideration:

1. Number of employees per unit and type (operator, technician, mechanic).

2. Number of packaging lines.

3. Result of simulation is a number or employees per type and per production unit allowed to change their shift.

Each production unit (PU) is evaluated independent (PU1, PU2 and PU3).

If PUs are considered completely separately, take PU1 as an example, let:

$x_{k, j}$ : indicates the number of type $j$ workers on the $k^{\text {th }}$ production line in PU1.

This means: 
$x_{k, 1}$ means the number of mechanics on the $k^{\text {th }}$ production line in PU1.

$x_{k, 2}$ means the number of operators on the $k^{\text {th }}$ production line in PU1.

$x_{k, 3}$ means the number of technicians on the $k^{\text {th }}$ production line in PU1.

Subject to:

Each production line requires a minimum of 0.5 technician, one mechanic and one operator to operate. The additional person can be either a mechanic or an operator or a technician as presented by Eq. (1):

$$
\sum_{k=1}^{8} x_{k, 1} \geq 8, \sum_{k=1}^{8} x_{k, 2} \geq 8 \text { and } \sum_{k=1}^{8} x_{k, 3} \geq 4
$$

and by Eq. (2) for each production line:

$$
\begin{gathered}
\sum_{j=1}^{3} x_{1, j} \geq 2.5, \sum_{j=1}^{3} x_{2, j} \geq 3.0, \sum_{j=1}^{3} x_{3, j} \geq 3.0, \sum_{j=1}^{3} x_{4, j} \geq 2.5, \sum_{j=1}^{3} x_{5, j} \geq 3.0, \\
\sum_{j=1}^{3} x_{6, j} \geq 2.5, \sum_{j=1}^{3} x_{7, j} \geq 2.5 \text { and } \sum_{j=1}^{3} x_{8, j} \geq 2.5
\end{gathered}
$$

Table III presents factory full capacity operation mode in corelation to minimum number of specific worker's type.

Table III: Factory full capacity per shift.

\begin{tabular}{|c|c|c|}
\hline Shift A & Shift B & Shift C \\
\hline$\sum_{k=1}^{8} x_{k, 1} \leq 10, \sum_{k=1}^{8} x_{k, 2} \leq$ & $\sum_{k=1}^{8} x_{k, 1} \leq 8, \sum_{k=1}^{8} x_{k, 2} \leq$ & $\sum_{k=1}^{8} x_{k, 1} \leq 11, \sum_{k=1}^{8} x_{k, 2} \leq$ \\
9 and $\sum_{k=1}^{8} x_{k, 3} \leq 6$ & 9 and $\sum_{k=1}^{8} x_{k, 3} \leq 5$ & 8 and $\sum_{k=1}^{8} x_{k, 3} \leq 5$ \\
\hline
\end{tabular}

Regarding the Eqs. (1) and (2) and factory full capacity per shift presented in Table III, the objective function can be defined by Eq. (3) as:

$$
f=\min _{x_{k, j}} \sum_{k=1}^{8} \sum_{j=1}^{3} x_{k, j}
$$

With the objective function, presented by Eq. (3), we can determinate the minimum number of workers required for each shift. That is, the remaining workers can choose the shift, if needed, or allocation by decision-making algorithm can be made. In general, we can say that everyone can choose his / her work shift, if the following conditions are met:

- take PU1 as an example, let:

$x_{k, 1, \mathrm{~A}}$ represents the number of mechanics who choose the shift A on the $k^{\text {th }}$ production line, $x_{k, 1, \mathrm{~B}}$ represents the number of mechanics who choose the shift $\mathrm{B}$ on the $k^{\text {th }}$ production line, $x_{k, 1, \mathrm{C}}$ represents the number of mechanics who choose the shift $\mathrm{C}$ on the $k^{\text {th }}$ production line, $x_{k, 2, \mathrm{~A}}$ represents the number of operators who choose the shift A on the $k^{\text {th }}$ production line, $x_{k, 2, \mathrm{~B}}$ represents the number of operators who choose the shift $\mathrm{B}$ on the $k^{\text {th }}$ production line, $x_{k, 2, \mathrm{C}}$ represents the number of operators who choose the shift $\mathrm{C}$ on the $k^{\text {th }}$ production line, $x_{k, 3, \mathrm{~A}}$ represents the number of technicians who choose the shift $\mathrm{A}$ on the $k^{\text {th }}$ production line, $x_{k, 3, \mathrm{~B}}$ represents the number of technicians who choose the shift $\mathrm{B}$ on the $k^{\text {th }}$ production line, $x_{k, 3, \mathrm{C}}$ represents the number of technicians who choose the shift $\mathrm{C}$ on the $k^{\text {th }}$ production line.

Table IIV: Workers schedule rotation per week.

\begin{tabular}{|c|c|c|c|c|}
\cline { 2 - 4 } \multicolumn{1}{c|}{} & Mechanics no. & Operators no. & Technicians no. & Week \\
\hline Shift A & M3 & O3 & T3 & \multirow{2}{*}{ First } \\
\hline Shift B & M1 & O1 & T1 & \\
\hline Shift C & M2 & O2 & T2 & \multirow{2}{*}{ Second } \\
\hline Shift A & M2 & O2 & T2 & \\
\hline Shift B & M3 & O3 & T3 & \multirow{2}{*}{ Third } \\
\hline Shift C & M1 & O1 & T1 & T1 \\
\hline Shift A & M1 & O1 & T2 & \\
\hline Shift B & M2 & O2 & T3 & \\
\hline Shift C & M3 & O3 & & \\
\hline
\end{tabular}


After initial workers schedule is made, we can propose long term schedule rotation plan for three weeks as presented in Table IV. Table IV presents general three weeks rotation plan in which real-time, daily workers allocation, must be allowed (taking into account, illness, free days, holiday, etc.).

\section{MATHEMATICAL FORMULATION OF THE MINIMUM NUMBER OF WORKERS REQUIRED PER SHIFT}

To develop a decision-making model for determining the optimal number of workers on production lines, a mathematical model has been created in which:

$x_{k, i, j}$ : indicates the number of type $j$ workers on the $k^{\text {th }}$ production line in PU $i$ per shift.

For example:

$x_{k, i, 1}$ : indicates the number of mechanics on the $k^{\text {th }}$ production line in PUi per shift.

$x_{k, i, 2}$ : indicates the number of operators on the $k^{\text {th }}$ production line in PUi per shift.

$x_{k, i, 3}$ : indicates the number of technicians on the $k^{\text {th }}$ production line in PU $i$ per shift.

$\ln _{i}$ : indicates the number of lines in PUi.

$\ln _{i, k}$ : indicates the number of workers required on line $k$ in PUi per shift.

Eqs. (4) and (5) present mathematical formulation for workers' per shift allocation:

$$
\begin{gathered}
\left\{\begin{array}{c}
x_{i, 1} \geq \ln _{i} \\
x_{i, 2} \geq \ln _{i} \\
x_{i, 3} \geq 0.5 \ln _{i}
\end{array}\right. \\
\sum_{j=1}^{3} x_{k, i, j} \geq \sum_{k=1}^{\ln l_{i}} \ln _{i, k}
\end{gathered}
$$

Mathematical formulation for real-world problem, where certain shift can be defined as:

$N M_{i}$ : indicates the number of mechanics in PUi of this certain shift.

$N O_{i}$ : indicates the number of operators in PUi of this certain shift.

$N T_{i}$ : indicates the number of technicians in PUi of this certain shift.

$N M C_{i}$ : indicates the number of mechanics who want to change their shift in PUi of this shift. $N O C_{i}$ : indicates the number of operators who want to change their shift in PUi of this shift. $N T C_{i}$ : indicates the number of technicians who want to change their shift in PUi of this shift.

If the following conditions, presented by Eq. (6), are met:

$$
\left\{\begin{array}{c}
N M_{i}-N M C_{i} \geq \ln _{i} \\
N O_{i}-N O C_{i} \geq \ln _{i} \\
N T_{i}-N T C_{i} \geq 0.5 \ln _{i} \\
\left(N M_{i}-N M C_{i}\right)+\left(N O_{i}-N O C_{i}\right)+\left(N T_{i}-N T C_{i}\right) \geq \sum_{k=1}^{l n_{i}} l_{i, k}
\end{array}\right.
$$

then the main objective function, for the production of this shift, of PUi, will not be affected (PU will obtain $100 \%$ productivity rate). If the above conditions are not met, the production will be affected, depending on how many workers of specific type are missing.

\section{ADVANCED DECISION-MAKING MATHEMATICAL MODEL}

In corelation to mathematical model presented in section three, an advanced decision-making mathematical model considering three shifts and different PU's is made, where:

$N W R L_{i, k}$ : represents the number of workers required on line $k$ in PUi per shift.

$N W_{i, j, s}$ : represents the number of type $j$ workers in shift $s$ of PUi in the current schedule.

$N W C_{i, j, s, d}$ : represents the number of type $j$ workers who want to change their shift from $s$ to $d$ in PUi in the next schedule (next week). 
$N W O_{i, j, s}$ : represents the number of type $j$ workers who want to change their shift $s$ for some other reason (for example, free time, holidays, sickness etc.) in PU $i$ in the next schedule.

$N W B_{i, j, s}$ : represents the number of type $j$ workers who want to return their shift $s$ after leaving for some other reason (for example, free time, holidays, sickness etc.) in PU $i$ in the next schedule (next week).

Note that for $j$ :

- $j=1$ : indicates the number of mechanics,

- $j=2$ : indicates the number of operators,

- $j=3$ : indicates the number of technicians.

For $s / d / o$ :

- $s / d / o=1$ : indicates the shift A,

- $s / d / o=2$ : indicates the shift $\mathrm{B}$,

- $s / d / o=3$ : indicates the shift $\mathrm{C}$.

Therefore, the number of workers of each type in each shift is determined by Eqs. (7) to (9):

$$
\begin{aligned}
& W_{i, 1, s}=N W_{i, 1, s}-\left(\sum_{d=1}^{3} N W C_{i, 1, s, d}+N W O_{i, 1, s}\right)+\left(\sum_{o=1}^{3} N W C_{i, 1, o, s}+N W B_{i, 1, s}\right) \\
& W_{i, 2, s}=N W_{i, 2, s}-\left(\sum_{d=1}^{3} N W C_{i, 2, s, d}+N W O_{i, 2, s}\right)+\left(\sum_{o=1}^{3} N W C_{i, 2, o, s}+N W B_{i, 2, s}\right) \\
& W_{i, 3, s}=N W_{i, 3, s}-\left(\sum_{d=1}^{3} N W C_{i, 3, s, d}+N W O_{i, 3, s}\right)+\left(\sum_{o=1}^{3} N W C_{i, 3, o, s}+N W B_{i, 3, s}\right)
\end{aligned}
$$

Still obtaining PU's $100 \%$ productivity rate, by meting the following conditions, presented by Eq. (10):

$$
\left\{\begin{array}{c}
W_{i, 1, s} \geq \ln _{i} \\
W_{i, 2, s} \geq \ln _{i} \\
W_{i, 3, s} \geq 0.5 \ln _{i} \\
\sum_{j=1}^{3} W_{i, j, s} \geq \sum_{j=1}^{\ln _{i}} \ln _{i, j}
\end{array}\right.
$$

\section{DESIGN OF EXPERIMENTS}

According to the mathematical model presented in section 4, Fig. 2 presents a block diagram of the decision-making algorithm. In the introductory phase, the input data of the PU's are obtained, to which constraints are assigned according to the target optimization function of ensuring the $100 \%$ operation mode of the produced lines and the appropriate number of workers scheduled. If the condition of providing the stated constraints and a sufficient number of input parameters and related capacities is met, the number of employees is recalculated according to the specific type of employee (mechanics, operator and technician). In the next step, the current state of the production system (current daily state of available workers) is checked again. This is followed by the implementation of the workers' allocation plan. If the workers allocation between PUs is made, this decision must be made by the PUs' shift managers.

The sequential operation of the algorithm is presented by Algorithm 1; the algorithm operates in a step loop in which it checks whether the available number of workers of a specific type is greater than or equal to the minimum PU's needs for workers, if this number is larger, algorithm schedules workers in the individual algorithm iteration, the algorithm subtracts the values from the available capacities and allocates them to the required production lines within the individual PU. When the algorithm detects a deviation from the available number of workers and minimum needs, the operation of the algorithm is stopped. If workers allocation is no longer possible (lack of resources/workers), the next steps are performed by a specific shift manager. In a case of workers' shortage, the algorithm shows the current (existing) PU's state and suggests possible partial solutions. 


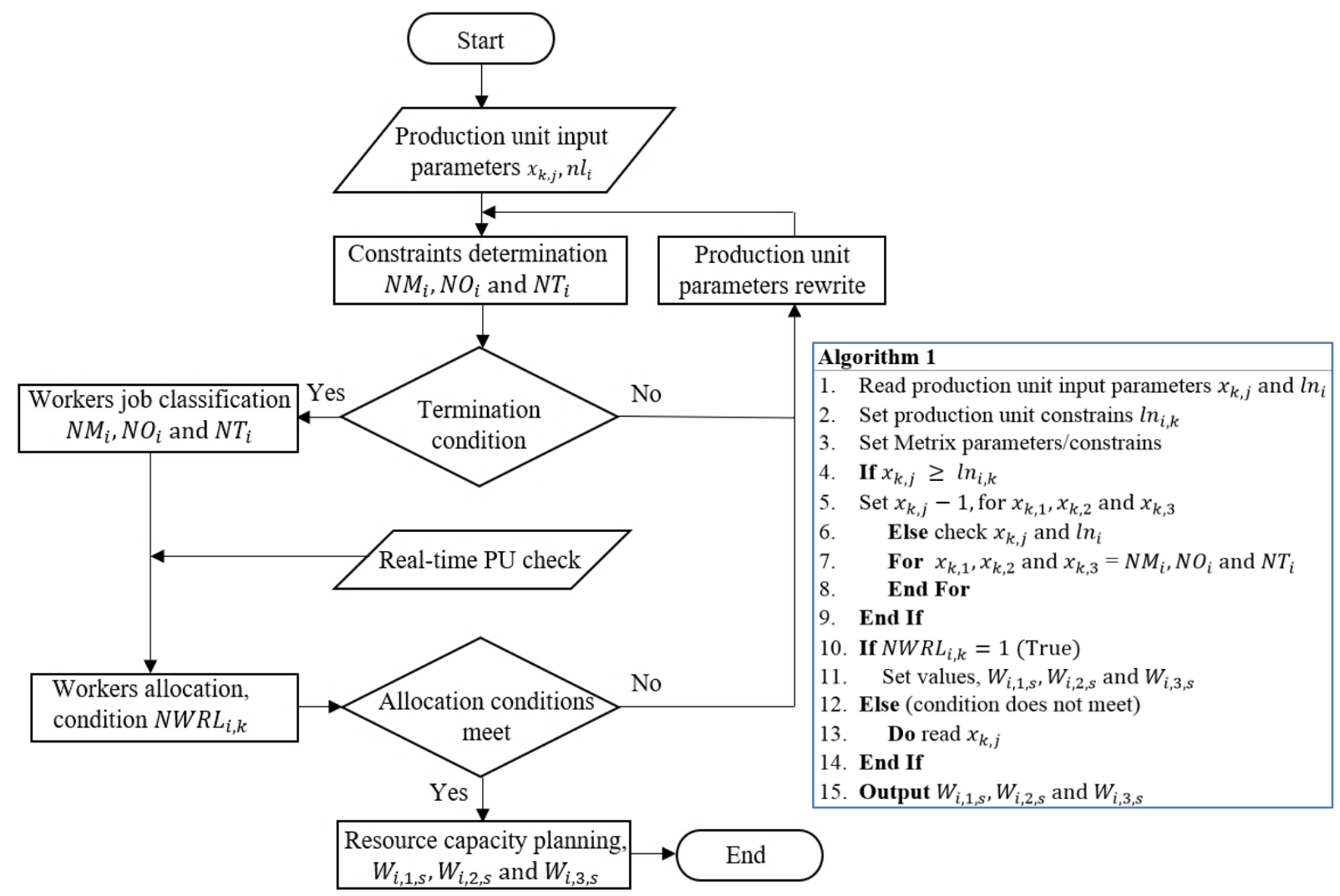

Figure 2: Algorithm block diagram structure (left) and its steps (right).

Table V shows the workers shifting between shifts according to the specific type of worker. Shift changes outside the initial scheduling plans may be made due to current absences or individual wishes, limitations and needs of employees. Dynamic (in this case) daily allocation of workers is extremely demanding for shift managers, as they must have different production capacities in mind. For this purpose, the algorithm itself, accordingly, proposes a solution or observations of irregularities that may occur in a specific dynamic event.

Table V: Workers shifting between shifts.

\begin{tabular}{|c|c|c|c|c|c|c|c|c|c|c|c|c|c|c|}
\hline \multicolumn{5}{|c|}{$N M_{i}$ need to change shift } & \multicolumn{5}{|c|}{$N O_{i}$ need to change shift } & \multicolumn{5}{|c|}{$N T_{i}$ need to change shift } \\
\hline From $\backslash$ To & A & B & $\mathrm{C}$ & $\sum$ & From $\backslash$ To & A & B & $\mathrm{C}$ & $\sum$ & From $\backslash$ To & A & B & $\mathrm{C}$ & $\sum$ \\
\hline $\mathrm{A}$ & & 2 & & 2 & $\mathrm{~A}$ & & & & 0 & $\mathrm{~A}$ & & & 2 & 2 \\
\hline B & & & 1 & 1 & B & 1 & & & 1 & B & & & & 0 \\
\hline $\mathrm{C}$ & 1 & & & 1 & $\mathrm{C}$ & & 1 & & 1 & $\mathrm{C}$ & 1 & & & 1 \\
\hline SUM & 1 & 2 & 1 & 4 & SUM & 1 & 1 & 0 & 2 & SUM & 1 & 0 & 2 & 3 \\
\hline
\end{tabular}

According to the dynamic changes shown in Table V, the algorithm shows the current PU's state (Table VI) and with the help of the decision-making model checks the adequacy of the number of workers according to the basic conditions of undistracted operation of the PU's.

Table VI: New workers schedule, with changes made.

\begin{tabular}{|c|c|c|c|c|}
\cline { 2 - 5 } \multicolumn{1}{c|}{} & $\boldsymbol{N M}_{\boldsymbol{i}}$ & $\boldsymbol{N O}_{\boldsymbol{i}}$ & $\boldsymbol{N T}_{\boldsymbol{i}}$ & $\sum$ \\
\hline Shift A & 9 & 14 & 6 & 29 \\
\hline Shift B & 9 & 14 & 3 & 26 \\
\hline Shift C & 10 & 7 & 7 & 24 \\
\hline$\sum$ & 28 & 35 & 16 & 79 \\
\hline
\end{tabular}


The basic PUs' constraint for workers allocation is that the $100 \%$ operation of production units must be performed. There are only two options, $100 \%$ operation, without interruptions, this option is considered by the algorithm as binary value 1 , or the lack of workers of specific type, which the algorithm considers as binary value 0 . Table VII shows binary values of adequacy of providing minimum number of workers according to required specific type correlated with individual shift. If the number of available workers of a specific type is less than the minimum required, the algorithm stops the workers' allocation operation and reports the inability of the production line to operate in a specific shift (Table VII red fields with a binary value of 0 ). However, if the scheduling and allocation plan of the workers was satisfied, given the $100 \%$ operation mode of the production lines, for an individual type of workers in a single shift, the binary value 1 is stated (Table VII, blue fields with a binary value of 1 ). The unwanted binary state 0 can lead to different daily dynamic changes inside PU's due to lack of workers. The main task of the algorithm in this case is to predict the limit value, how many dynamic changes (workers individual daily allocations) can happen that the system will run smoothly (Table VII, green fields with a binary value 1). Final determination of limit values proposed by the algorithm is adopted by the shift manager. The decision algorithm shows the current state of PU's and available capacities of workers.

Table VII: Algorithm allocation results: minimum number of workers check.

\begin{tabular}{|c|c|c|c|c|c|}
\cline { 2 - 6 } \multicolumn{1}{c|}{} & $\boldsymbol{N M}_{\boldsymbol{i}}$ & $\boldsymbol{N O}_{\boldsymbol{i}}$ & $\boldsymbol{N T}_{\boldsymbol{i}}$ & $\sum$ & Minimum number of workers \\
\hline Shift A & 0 & 1 & 0 & 1 & 0 \\
\hline Shift B & 1 & 1 & 1 & 3 & 1 \\
\hline Shift C & 1 & 0 & 1 & 2 & 0 \\
\hline$\sum$ & 2 & 2 & 2 & 6 & 1 \\
\hline
\end{tabular}

\section{RESULTS AND DISCUSSION}

As a result of the decision-making model for determining the optimal number of workers with constraints, the allocation plan of workers (in Table VIII for the mechanics) for the three-week rotation cycle is presented. In the column "Worker no." are named workers of a specific type and a shift allocation in which they work. In the "State" column, the algorithm shows the current state of workers in the PU taking into account the dynamic changes. The stated values represent the minimum number of required workers of a specific type, and the boldly marked values are positive or negative values of available workers of a specific type in an individual shift. The specified value is calculated by the algorithm, and the final shift decision is made by the shift manager. When the shift manager makes a change, he/she enters changes into the system and the algorithm recalculates the workers allocation plan including the new state and any new dynamic changes. Table VIII presents only the part with mechanics for PU1, otherwise the shift manager operates with the entire allocation plan of workers (mechanics, operators and technicians) related to the individual shift and PU.

As described, Table VIII shows the workers' allocation plan for the three-week rotation cycle, which is used daily and inspected by the shift manager within the individual PUs. However, since companies often need a long-term plan (training plan, educational plan and new employments) not only in a specific PU, the algorithm constantly recalculates and displays the results for the entire production system, in this case for three PUs. Based on the results shown in Table IX, the company's long-term planning and scheduling capacities can consequently eliminate shortcomings. Based on the presented results, the company can plan the movements of workers between the PUs and thus perform more efficiently with the available capacity. With the help of an appropriate education plan, they can successfully lead a strategy of promotions, training, and eligible new jobs. 
Table VIII: Long term scheduling results.

\begin{tabular}{|c|c|c|c|c|c|c|c|c|}
\hline \multicolumn{2}{|c|}{ Week 1 } & \multirow{2}{*}{ State } & \multicolumn{2}{c|}{ Week 2 } & \multirow{2}{*}{ State } & \multicolumn{2}{c|}{ Week 3 } & \multirow{2}{*}{ State } \\
\cline { 1 - 1 } Worker no. & Shift & & Worker no. & Shift & & Worker no. & Shift & \\
\hline M01 & A & $N M_{i}$ in shift A & M01 & B & $N M_{i}$ in shift A & M01 & C & $N M_{i}$ in shift A \\
\hline M02 & A & 10 & M02 & B & 11 & M02 & C & 9 \\
\hline M03 & A & $\mathbf{0}$ & M03 & B & $\mathbf{1}$ & M03 & C & -1 \\
\hline M04 & A & $N M_{i}$ in shift B & M04 & B & $N M_{i}$ in shift B & M04 & C & $N M_{i}$ in shift B \\
\hline M05 & A & 9 & M05 & B & 10 & M05 & C & 10 \\
\hline M06 & A & $\mathbf{- 1}$ & M06 & B & $\mathbf{0}$ & M06 & C & 0 \\
\hline M07 & A & $N M_{i}$ in shift C & M07 & B & $N M_{i}$ in shift C & M07 & C & $N M_{i}$ in shift C \\
\hline M08 & A & 10 & M08 & B & 8 & M08 & C & 10 \\
\hline M09 & A & $\mathbf{0}$ & M09 & B & $-\mathbf{2}$ & M09 & C & 0 \\
\hline
\end{tabular}

Table IVIII: Production system state.

\begin{tabular}{|c|c|c|c|c|}
\hline Production unit & Shift & $\boldsymbol{N M}_{\boldsymbol{i}}$ reguired & $\boldsymbol{N M}_{\boldsymbol{i}}$ courent state & $\sum$ \\
\hline \multirow{3}{*}{ PU1 } & Shift A & 10 & 10 & $\mathbf{0}$ \\
\cline { 2 - 5 } & Shift B & 10 & 8 & $\mathbf{- 2}$ \\
\cline { 2 - 5 } & Shift C & 10 & 11 & $\mathbf{1}$ \\
\hline \multirow{3}{*}{ PU2 } & Shift A & 10 & 9 & $\mathbf{- 1}$ \\
\cline { 2 - 5 } & Shift B & 10 & 10 & $\mathbf{0}$ \\
\cline { 2 - 5 } & Shift C & 10 & 8 & $\mathbf{- 2}$ \\
\hline
\end{tabular}

\section{CONCLUSION}

The initial research question regarding the importance of efficient RCP of workers with different constraints for certain tasks and at the same time ensuring continuous (undisrupted) operation of the production lines is presented in the research work, with the new decisionmaking algorithm that is able to perform RCP. The results show that the presented decisionmaking model is able to schedule different types of workers (mechanics, operators, technicians) with different constraints (shift rotation, free day, etc.) on specific production line while ensuring the smooth operation of the production system. The advantage of the presented mathematical model is the advanced RCP of workers, which includes various constraints and assumptions, while the program is able to perform long- and short-term workers' allocation, ensuring so-called ability of a dynamic RCP. In the current phase of the research, the algorithm focuses on workers allocation under the condition that the production system is $100 \%$ utilized. There are several types of production systems in practice, where such a mode of operation is purely theoretical and cannot be implemented. To this end, in addition to the mathematical model, the presented research work provides an algorithm and the corresponding pseudocode intended for the general scheduling of workers under various constraints, regardless of the production system type. The presented numerically simulated experiments show how we can optimize the changes made due to unannounced workers changes in real-time (daily, shift changes) while ensuring the smooth operation of the line. The proposed decision-making algorithm suggests a solution to the decision-makers (production unit or shift managers) in a case of impossibility of optimal allocation. The presented results prove the time and costefficient allocation of workers ensuring undisturbed operation of the production system, while allowing the company to adapt to the demand and resources of workers in the production ensuring efficient additional training, education, and new employments.

Further research will be based on the extension of the decision-making model, which is intended for a wider range of production systems and various optimization parameters. However, even if the optimal workers' allocation schedule is not possible, the algorithm will propose and decide the current suboptimal schedule. This is especially important when dealing 
with the acquisition and processing a large amount of data (numerical, graphical), then the physical decision-makers have difficulty in selecting /judging/making the optimal solution.

\section{ACKNOWLEDGEMENT}

This research was funded by the Slovenian Research Agency (ARRS), Research Core Grant P2-0190.

\section{REFERENCES}

[1] Yura, K. (1994). Production scheduling to satisfy worker's preferences for days off and overtime under due-date constraints, International Journal of Production Economics, Vol. 33, No. 1-3, 265270, doi:10.1016/0925-5273(94)90139-2

[2] Dukić Mijatović, M.; Uzelac, O.; Stoiljković, A. (2020). Effects of human resources management on the manufacturing firm performance: Sustainable development approach, International Journal of Industrial Engineering and Management, Vol. 11, No. 3, 205-212, doi:10.24867/IJIEM-20203-265

[3] Ojstersek, R.; Tang, M.; Buchmeister, B. (2020). Due date optimization in multi-objective scheduling of flexible job shop production, Advances in Production Engineering \& Management, Vol. 15, No. 4, 481-492, doi:10.14743/APEM2020.4.380

[4] Legato, P.; Mazza, R. M. (2001). Berth planning and resources optimisation at a container terminal via discrete event simulation, European Journal of Operational Research, Vol. 133, No. 3, $537-$ 547, doi:10.1016/S0377-2217(00)00200-9

[5] Golden, L. (2001). Flexible work schedules: Which workers get them?, American Behavioral Scientist, Vol. 44, No. 7, 1157-1178, doi:10.1177/00027640121956700

[6] Ojstersek, R.; Acko, B.; Buchmeister, B. (2020). Simulation study of a flexible manufacturing system regarding sustainability, International Journal of Simulation Modelling, Vol. 19, No. 1, 6576, doi:10.2507/IJSIMM19-1-502

[7] Park, J.-H.; Suh, H.-J.; Yang, H.-D. (2007). Perceived absorptive capacity of individual users in performance of Enterprise Resource Planning (ERP) usage: The case for Korean firms, Information \& Management, Vol. 44, No. 3, 300-312, doi:10.1016/j.im.2007.02.001

[8] Mark, B. G.; Hofmayer, S.; Rauch, E.; Matt, D. T. (2019). Inclusion of workers with disabilities in production 4.0: Legal foundations in Europe and potentials through worker assistance systems, Sustainability, Vol. 11, No. 21, Paper 5978, 21 pages, doi:10.3390/su11215978

[9] Chaudhry, I. A.; Drake, P. R. (2009). Minimizing total tardiness for the machine scheduling and worker assignment problems in identical parallel machines using genetic algorithms, The International Journal of Advanced Manufacturing Technology, Vol. 42, No. 5-6, 581-594, doi:10.1007/s00170-008-1617-z

[10] Campbell, G. M. (2011). A two-stage stochastic program for scheduling and allocating crosstrained workers, Journal of the Operational Research Society, Vol. 62, No. 6, 1038-1047, doi:10.1057/jors.2010.16

[11] Moreira, M. C. O.; Costa, A. M. (2013). Hybrid heuristics for planning job rotation schedules in assembly lines with heterogeneous workers, International Journal of Production Economics, Vol. 141, No. 2, 552-560, doi:10.1016/j.ijpe.2012.09.011

[12] Zeinali, F.; Mahootchi, M.; Sepehri, M. M. (2015). Resource planning in the emergency departments: A simulation-based metamodeling approach, Simulation Modelling Practice and Theory, Vol. 53, 123-138, doi:10.1016/j.simpat.2015.02.002

[13] Benavides, A. J.; Ritt, M.; Miralles, C. (2014). Flow shop scheduling with heterogeneous workers, European Journal of Operational Research, Vol. 237, No. 2, 713-720, doi:10.1016/ j.ejor.2014.02.012

[14] Xu, Z.; Ming, X. G.; Zheng, M.; Li, M.; He, L.; Song, W. (2015). Cross-trained workers scheduling for field service using improved NSGA-II, International Journal of Production Research, Vol. 53, No. 4, 1255-1272, doi:10.1080/00207543.2014.955923

[15] Tabataba'i Nasab, S. M.; Kaveh, M. (2016). A parallel simulated annealing (PSA) for solving project scheduling problem with discounted cash flow policy in pricing strategy of the project suppliers, Technical Gazette, Vol. 23, No. 6, 1555-1563, doi:10.17559/TV-20140425121514 
[16] Zhao, X. F.; Liu, H. Z.; Lin, S. X.; Chen, Y. K. (2020). Design and implementation of a multiple AGV scheduling algorithm for a job-shop, International Journal of Simulation Modelling, Vol. 19, No. 1, 134-145, doi:10.2507/IJSIMM19-1-CO2

[17] Berthelsen, M.; Pallesen, S.; Bjorvatn, B.; Knardahl, S. (2015). Shift schedules, work factors, and mental health among onshore and offshore workers in the Norwegian petroleum industry, Industrial Health, Vol. 53, No. 3, 280-292, doi:10.2486/indhealth.2014-0186

[18] Lohmann, M.; Anzanello, M. J.; Fogliatto, F. S.; da Silveira, G. C. (2019). Grouping workers with similar learning profiles in mass customization production lines, Computers \& Industrial Engineering, Vol. 131, 542-551, doi:10.1016/j.cie.2019.03.012

[19] Katić, I.; Berber, N.; Slavić, A.; Ivanišević, A. (2020). The relations between investment in employees' development and organizational productivity and service quality, Technical Gazette, Vol. 27, No. 4, 1077-1083, doi:10.17559/TV-20181121101314

[20] Rahmani, D.; Ramezanian, R. (2016). A stable reactive approach in dynamic flexible flow shop scheduling with unexpected disruptions: A case study, Computers \& Industrial Engineering, Vol. 98, 360-372, doi:10.1016/j.cie.2016.06.018

[21] Hozdić, E.; Kozjek, D.; Butala, P. (2020). A cyber-physical approach to the management and control of manufacturing systems, Strojniski vestnik - Journal of Mechanical Engineering, Vol. 66, No. 1, 61-70, doi:10.5545/sv-jme.2019.6156

[22] Zheng, Z. L.; Bao, X. (2019). The investment strategy and capacity portfolio optimization in the supply chain with spillover effect based on artificial fish swarm algorithm, Advances in Production Engineering \& Management, Vol. 14, No. 2, 239-250, doi:10.14743/APEM2019.2.325

[23] Freile, A. J.; Mula, J.; Campuzano-Bolarin, F. (2020). Integrating inventory and transport capacity planning in a food supply chain, International Journal of Simulation Modelling, Vol. 19, No. 3, 434-445, doi:10.2507/IJSIMM19-3-523

[24] Trstenjak, M.; Ćosić, P.; Antolić, D. (2019). Workpiece classification criteria in automated process planning, Technical Gazette, Vol. 26, No. 1, 256-262, doi:10.17559/TV-20180215105405

[25] Akbari, M.; Zandieh, M.; Dorri, B. (2013). Scheduling part-time and mixed-skilled workers to maximize employee satisfaction, The International Journal of Advanced Manufacturing Technology, Vol. 64, No. 5, 1017-1027, doi:10.1007/s00170-012-4032-4 